\title{
Inhibition of Lipoxygenases Showed No Benefit for the Musculoskeletal System in Estrogen Deficient Rats
}

\section{OPEN ACCESS \\ Edited by:}

Melissa Orlandin Premaor, Federal University of Minas Gerais,

Brazil

Reviewed by:

Astrid Kamilla Stunes, Norwegian University of Science and Technology, Norway Hitoshi Saito,

Chugai Pharmaceutical Co., Ltd, Japan

*Correspondence: Dominik Saul Dominik.Saul@med.uni-goettingen.de

tORCID:

Dominik Saul orcid.org/0000-0002-0673-3710 Marina Komrakova orcid.org/0000-0002-6225-4378

Specialty section: This article was submitted to Bone Research, a section of the journal Frontiers in Endocrinology

Received: 07 May 2021 Accepted: 29 June 2021 Published: 20 July 2021

Citation:

Saul D, Hohl FE, Franz MK, Meyer I, Taudien S, Roch PJ, Sehmisch S and Komrakova M (2021) Inhibition of Lipoxygenases Showed No Benefit for the Musculoskeletal System in Estrogen Deficient Rats.

Front. Endocrinol. 12:706504. doi: 10.3389/fendo.2021.706504

\begin{abstract}
Dominik Saul ${ }^{1,2 *}{ }^{2}$, Friederike Eva Hohl ${ }^{1}$, Max Konrad Franz ${ }^{1}$, llka Meyer ${ }^{1}$, Stefan Taudien ${ }^{3}$, Paul Jonathan Roch ${ }^{1}$, Stephan Sehmisch ${ }^{1}$ and Marina Komrakova ${ }^{1 \dagger}$

${ }^{1}$ Department of Trauma Surgery, Orthopaedics and Plastic Surgery, University Medical Center Goettingen, Goettingen, Germany, ${ }^{2}$ Kogod Center on Aging and Division of Endocrinology, Mayo Clinic, Rochester, MN, United States, ${ }^{3}$ Division of Infection Control and Infectious Diseases, Georg-August-University of Goettingen, Goettingen, Germany
\end{abstract}

Background: In previous studies, we reported the beneficial impact of two lipoxygenaseinhibitors, Baicalein and Zileuton, on osteoporotic bone in a postmenopausal rat model. Whereas subcutaneous Baicalein predominantly improved cortical bone, Zileuton enhanced vertebral and femoral trabecular bone. In this study, we aimed to reveal whether the oral administration of Baicalein caused similar effects on bone and whether a combined administration of Baicalein and Zileuton could act synergistically to ameliorate the formerly reported effects in the musculoskeletal system.

Methods: We treated ovariectomized (OVX) female Sprague-Dawley rats either with Baicalein (10mg/kg BW), Zileuton (10 mg/kg BW) or a combination of both (each 10mg/kg BW) for 13 weeks and compared with untreated OVX and NON-OVX groups ( $n=12-16$ rats per group). Lumbar vertebral bodies and femora were analyzed. Tibiae were osteotomized, plate-stabilized (at week 8 after OVX) and likewise analyzed by biomechanical, histological, micro-computed tomographical and ashing tests. The skeletal muscle structure was analyzed.

Results: Oral administration of Baicalein did not confirm the reported favorable cortical effects in neither vertebra nor femur. Zileuton showed a beneficial effect on trabecular vertebra, while the femur was negatively affected. Callus formation was enhanced by all treatments; however, its density and biomechanical properties were unaltered. Lipoxygenase inhibition did not show a beneficial effect on skeletal muscle. The combination therapy did not ameliorate OVX-induced osteoporosis but induced even more bone loss.

Conclusions: The preventive anti-osteoporotic treatments with two lipoxygenase inhibitors applied either alone or in combination showed no benefit for the musculoskeletal system in estrogen deficient rats.

Keywords: baicalein, bone healing, lipoxygenase-inhibitor, muscle tissue, osteoporosis, zileuton 


\section{INTRODUCTION}

With a prevalence of 200 million people worldwide, mostly postmenopausal women are affected by osteopenia or osteoporosis. In line with that, the annual amount of osteoporotic fractures among Medicare beneficiaries in the United States is estimated to be over 2.3 million (1-3). The "silent disease" loses covertness more and more due to extended guidelines and better prevention, but yet the long-term use of established medicamentous therapies causes several new dilemmas (4). Consequently and with a better understanding of underlying mechanisms that control bone turnover, new targets of therapy have been discovered and addressed (5). On this way, Receptor Activator of NF- $\kappa B$ Ligand (RANKL)- and Sclerostin-antibodies have found their way into the clinic $(6,7)$. However, advanced search for novel therapeutic approaches is ongoing.

Lipoxygenase (LOX) inhibitors have been found to suppress osteoclast formation and enhance bone formation in vivo $(8,9)$. A LOX inhibitor, Baicalein, which is extracted from the plant Scutellaria baicalensis inhibits 12- and 15-Lipoxygenase (12- and 15-LOX). Another lipoxygenase inhibitor, Zileuton, inhibits 5LOX and has been FDA-approved $\left(\mathrm{Zyflo}^{\circledR}\right)$ for the treatment of asthma. Both lipoxygenase-inhibitors exert an antioxidative effect providing protection for cellular components and inhibiting apoptosis both in in vitro and in vivo studies (10-12).

In our previous studies we reported beneficial effects of these two LOX inhibitors applied as a mono-therapy for 4 to 5 weeks on bone parameters in the postmenopausal rat model. Baicalein was able to improve cortical bone, while Zileuton enhanced the trabecular structure in lumbar vertebrae and femora $(13,14)$. In addition to that, we demonstrated a beneficial effect of these treatments on the capillarization of the skeletal muscle (15). The bone healing process was also favorably affected by these LOX inhibitors $(14,16)$. Zileuton therapy improved cortical, callus and total bone volume at the osteotomy site in the tibia in ovariectomized rats (14), while a Baicalein monotherapy enhanced callus density without improving callus formation rate (16).

In the present study, we subsequently aimed to examine whether a combined therapy of Zileuton and Baicalein had an additional or interfering effect on cortical and trabecular bone in the lumbar spine and femur in an estrogen-deficient rat model. Furthermore, we elucidated the bone healing process in the tibia amongst these treatments and investigated their effects on skeletal muscle tissue. The LOX-inhibitors were applied orally to overcome the negative side effects of subcutaneous Baicalein administration (15) for a prolonged period of thirteen weeks, after the pharmacokinetics of Baicalein, orally administered in a concentration of 10 and $20 \mathrm{mg} / \mathrm{kg}$, have been demonstrated to be reliably stable in rats by Kim et al. (17).

\section{MATERIAL AND METHODS}

\section{Animals and Treatment}

Seventy virgin female Sprague-Dawley rats (Janvier, Le GenestSaint-Isle, France) were housed at $20^{\circ} \mathrm{C}$ and with a humidity of
$55 \%$ in Makrolon ${ }^{\circledR}$ IV cages (Techniplast Germany GmbH, Hohenpeissenberg, Germany). The experiments were conducted after approval of the local district government and in compliance with the ethical standards of animal care (no. 14/1530).

The rats were ovariectomized at the age of 3 months, one group $(\mathrm{n}=12)$ was left non-ovariectomized (NON-OVX). OVX rats were randomly divided into 4 groups and treated orally according to the group assignment for up to 13 weeks. One group was ovariectomized and left untreated (OVX, $n=12$ ), while another group was ovariectomized and received oral Baicalein therapy $(10 \mathrm{mg} / \mathrm{kg}$ body weight $[\mathrm{BW}]$, Baicalein, $\mathrm{n}=15)$. The Zileuton-group was ovariectomized and received $10 \mathrm{mg} / \mathrm{kg} \mathrm{BW}$ of Zileuton (Zileuton, $\mathrm{n}=15$ ) and the other ovariectomized group received both Baicalein and Zileuton (each $10 \mathrm{mg} / \mathrm{kg} \mathrm{BW}$, Baicalein+Zileuton, $n=16$, Figure 1A).

A bilateral ovariectomy was performed as described earlier (13). Briefly, the surgical procedure was carried out under isoflurane anesthesia. Accordingly, anesthesia, shaving and disinfection were followed by incision on the abdomen bilaterally and dissection of adnexa before wound closure. Immediately after OVX, treatments with Baicalein and/or Zileuton were started. A soy-free diet $\left[\right.$ ssniff $^{\circledR}$ special diet; $\mathrm{GmbH}$, Soest, Germany, ingredients listed in (13)] was supplemented either with Zileuton (Zyflo ${ }^{\circledR}$; Cornerstone Therapeutics Inc., Cary, NC, USA) and/or Baicalein (98\%; Sigma-Aldrich Chemie GmbH, Munich, Germany) at a concentration of $175 \mathrm{mg} / \mathrm{kg}$ food to achieve the dose of $10 \mathrm{mg} /$ $\mathrm{kg}$ BW, respectively. This dose of Baicalein and Zileuton was shown to have an effect on bone tissue in previous studies (13$15,18)$. During the experiment, all rats received soy-free diet either supplemented with Baicalein and/or Zileuton or without supplementation and demineralized water ad libitum.

Body weight and food intake were recorded on a weekly basis, with calculation of average daily food intake. The resulting effective dose averaged $10.35 \pm 0.24 \mathrm{mg} / \mathrm{kg}$ BW in Baicalein group, $10.43 \pm 0.22 \mathrm{mg} / \mathrm{kg} \mathrm{BW}$ in Zileuton group and $10.48 \pm$ $0.26 \mathrm{mg} / \mathrm{kg} \mathrm{BW}$ (both) in Baicalein+Zileuton group. To ensure comparable amounts of food uptake, the individual rat weight was measured on a weekly basis (Figures 1B, C).

Eight weeks after OVX, a bilateral $0.5 \mathrm{~mm}$ osteotomy of the tibia metaphysis with consecutive 5-hole T-shaped titanium plate osteosynthesis (57-05140; Stryker Trauma, Selzach, Switzerland) was performed as described earlier (19) (Figure 2F). The osteotomy operation was performed under intraperitoneal anesthesia with $38 \mathrm{mg}$ ketamine (Ketamin Inresa, Inresa Arzneimittel $\mathrm{GmbH}$, Freiburg, Germany) and $2.5 \mathrm{mg}$ midazolam (Rotexmedica GmbH, Trittau, Germany) per kg $\mathrm{BW}$, respectively, along with isoflurane inhalation anesthesia $(14,19)$. Four rats died during osteotomy operation due to an anaesthesia side effect. After the osteotomy, rats were treated with $5 \mathrm{mg} / \mathrm{kg}$ BW Carprieve (Carprieve ${ }^{\circledR} 50 \mathrm{mgl} / \mathrm{ml}$ Injekt, Norbrook Labs Ltd, Newry, Northern Ireland) twice a day for 5 days. Thereafter, 16 rats died due to the perforation of the intestine and the pain therapy was interrupted. No further losses were observed. Thus, precautions should be taken by applying 

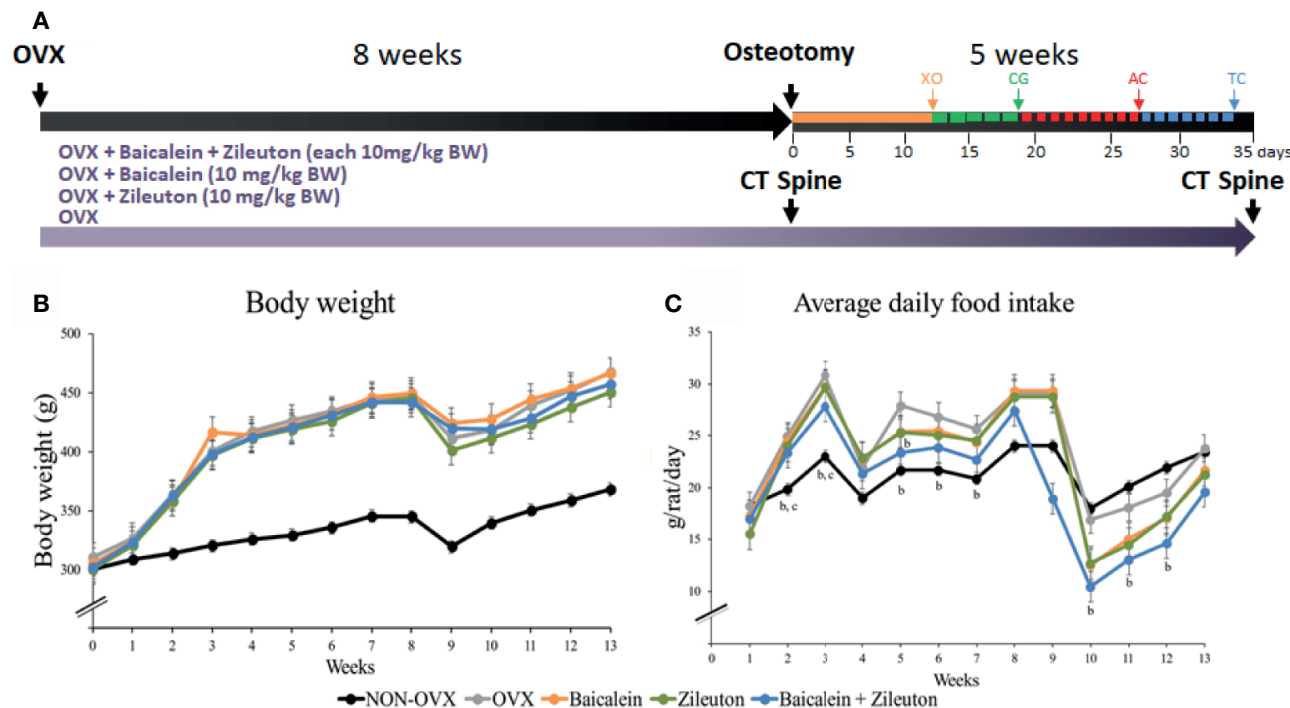

FIGURE 1 Graphical abstract of experimental setup. (A) After ovariectomy, 13 weeks-treatments with either Baicalein, Zileuton or their combination were conducted. Bilateral tibia osteotomy was performed 8 weeks after OVX. In vivo CT of the spine was executed after 8 and 13 weeks. On day 12 , xylenol orange was administered, on day 19 calcein green, on day 27 alizarin complexone, followed by tetracycline on day 33. (B) The body weight of rats (g). (C) The average daily food intake (g/rat/day). ${ }^{b}$ Differs from OVX. ${ }^{\circ}$ Differs from Baicalein.

analgesic treatments, since one of the side effects of NSAIDs is gastrointestinal damage (20). The administration of Carprieve once per day proved to be sufficient and had no severe side effects $(19,21,22)$.

At the end of the experiment, the following numbers of animals were analyzed in NON-OVX group: 8, in OVX: 10, in Baicalein: 11, in Zileuton: 11 and in Baicalein+Zileuton 10 .

Thirteen weeks after OVX, the rats were weighed, and decapitated after $\mathrm{CO}_{2}$-anesthesia. The uterus weight was recorded. Forth lumbar vertebral body (L4) was isolated, stored at $-20^{\circ} \mathrm{C}$ for micro-computed tomography (micro-CT), ashing and compression test as described earlier (13). Right femora and right osteotomized tibiae were stored at $-20^{\circ} \mathrm{C}$ for biomechanical, ashing and micro-CT analyses (23).

\section{In Vivo Quantitative Computed Tomography}

Eight and thirteen weeks after OVX, in vivo pQCT of L4 was performed in isoflurane-anesthetized rats ( $n=5 /$ group) using a pQCT device XCT Research SA (Stratec Medizintechnik GmbH, Pforzheim, Germany). The scans were performed in the same rats at both time points, with an exception in groups NON-OVX, OVX and Baicalein + Zileuton, where one rat in each group was replaced because of losses occurred after osteotomy. Bone mineral density $\left(\mathrm{BMD}, \mathrm{mg} / \mathrm{cm}^{3}\right)$, total bone area $\left(\mathrm{mm}^{2}\right)$ and stress-strain index (SSI) of L4 (central slice) and cross-sectional abdominal area $\left(\mathrm{CSA}, \mathrm{mm}^{2}\right)$ were measured as described in detail earlier (21). Three digital sections were analyzed for each animal. Analysis was performed using XCT-6.20C software (Stratec Medizintechnik GmbH, Pforzheim, Germany) as described earlier (13).

\section{Biomechanical Test}

Biomechanical parameters of L4, right femora and right tibiae were assessed with a Zwick machine (145,660 Z020/TND; Ulm, Germany) (22-24). The L4 was fixed at the aluminum base and the stamp was loaded to the vertebral body (compression test). The femoral head was located in a $4 \mathrm{~mm}$ well on the end of the plate and loaded to the trochanteric region (breaking test). The tibia was placed on the aluminum base and loaded $2-3 \mathrm{~mm}$ distally to the osteotomy at tuberositas tibiae (bending test). A stamp mechanically dropped with $5 \mathrm{~mm} / \mathrm{min}$ onto the bone with an initial force of $1 \mathrm{~N}$. After this fixing procedure, measurements were performed with an accuracy of $0.2-0.4 \%$ within 2 and $500 \mathrm{~N}$ and recorded with the aid of testXpert software (Zwick GmbH \& Co. KG, Ulm, Germany) (13). The test was finalized after the initial linear curve increase declined more than $10 \mathrm{~N}$ in $\mathrm{L} 4$ and femur and more than $2 \mathrm{~N}$ in tibia. In femur, the test was performed until fracture occurred, whereas in tibia until plastic deformation of the bone. Reported values were the highest force, that the bone could withstand (Fmax, N) and slope of linear increment of the curve (Stiffness, N/mm), calculated using Microsoft Excel (Microsoft Corporation, Redmond, WA) (13).

\section{Micro-CT Analysis}

To analyze cortical and trabecular parameters, L4, right femora and right tibiae were scanned using Quantum FX micro-CT (Caliper Sciences, Hopkinton, MA, USA). The scan protocol was as follows: $70 \mathrm{kVp}, 200 \mu \mathrm{A}, 30 \mathrm{~Hz}$ detector frame rate, $360^{\circ}$ rotation, 3600 projections, $20 \times 20 \mathrm{~mm}^{2}$ field of view, 512-pixel matrix and a $40 \mu \mathrm{m}$ resolution. A phantom block with 5 defined hydroxyapatite elements $\left(0.2,0.4,0.6,0.8\right.$ and $\left.1.0 \mathrm{~g} / \mathrm{cm}^{3}\right)$ accompanied every scan to convert grey values into bone 
mineral density (BMD) (16). 3D OsteoAnalyze software was used to calculate density and volume (16). Femoral region-ofinterest was the head, which was cut in the transition zone from the collum femoris to the trochanter major. BV/TV, total BMD, cortical BMD (Ct.BMD), trabecular BMD (Tb.BMD), total and soft tissue volumes were measured $(13,25)$. Vertebral region-ofinterest was the corpus vertebra as described earlier. Herein, we analyzed trabecular BMD (Tb.BMD), trabecular volume (Tb.V), total BMD and BV/TV (13). Tibial region-of-interest extended $1.5 \mathrm{~mm}$ proximally and distally from the osteotomy line (14). Analyzed values were cortical BMD (Ct.BMD), cortical volume (Ct.V), total bone BMD, total callus BMD (Cl.BMD) and osseous callus volume fraction (osseous $\mathrm{Cl}$. V/total $\mathrm{Cl} . \mathrm{V}$ ) according to Bouxsein et al. (26). An exemplarily representation of the used thresholds with a NON-OVX rat for femur, tibia and lumbar vertebral body is shown in Supplementary Figure 1.

Further detailed structural analyses were performed on transformed 2D images. Three images of sagittal cut vertebral bodies, femora and tibiae were analyzed using MetaMorph Basic Acquisition Software (Leica Mikrosysteme Vertrieb GmbH, Wetzlar, Germany). Collected cortical data were thickness (Ct.Th, $\mathrm{mm})$, area (Ct.Ar, $\mathrm{mm}^{2}$ ) and density (Ct. Dn, \%). Trabecular parameters were density (Tb.Dn, \%), number of nodes (N.Nd), density of trabecular nodes $\left(\mathrm{N} / \mathrm{mm}^{2}\right)$, trabecular thickness (Tb.Th, $\mathrm{mm})$ and trabecular area ( $\left.\mathrm{Tb} . \mathrm{Ar}, \mathrm{mm}^{2}\right)$. Callus parameters were density (Cl. Dn, \%) and thickness $(\mathrm{Cl}$. Th, mm) $(13,14,16)$.

\section{Bone Healing Analysis}

Dynamic callus formation was monitored using histological sections of right tibia stained in-vivo with fluorescent dyes: Xylenol orange (XO, $90 \mathrm{mg} / \mathrm{kg} \mathrm{BW})$, calcein green (CG, 10 $\mathrm{mg} / \mathrm{kg} \mathrm{BW}$ ), alizarin complexone (AC, 30mg/kg BW) and tetracycline (TC, $25 \mathrm{mg} / \mathrm{kg} \mathrm{BW}$ ) were injected subcutaneously on days 12, 19, 27 and 33 after osteotomy, respectively (16) (Figure 1A). Briefly, tibia samples embedded in methyl methacrylate (Merck, Darmstadt, Germany) were cut longitudinally (150 $\mu \mathrm{m}$ thickness) using a diamond saw microtome (Leica SP1600, Leica Instruments $\mathrm{GmbH}$, Nussloch, Germany). Subsequently, the 3 representative central sections were digitalized using a digital camera (Leica DC300F) and a zoom stereo microscope (Leica MZ75, Bensheim, Germany). The measurement area extended $2.5 \mathrm{~mm}$ from the osteotomy line and was divided into a ventral (plate side), dorsal (opposite side) and endosteal part. In each of these, the total callus area and labelingspecific callus areas were determined using the MetaMorph Basic Acquisition Software (Leica Mikrosysteme). XO and CG labeled callus areas were measured concurrently (XO+CG) (22).

\section{Ashing}

For analysis of mineral content, L4 and right femur were ashed at $750^{\circ} \mathrm{C}$ for one hour in a muffle oven. Weight before ashing (organic) and after ashing (anorganic) were recorded $(13,27)$.

\section{Muscle Analysis}

After sacrifice, Musculus gastrocnemius, soleus and longissimus were extracted and weighed. All muscles were frozen in liquid nitrogen and stored at $-80^{\circ} \mathrm{C}$ for further histological analyses.
Muscle from the right side were used for histological analyses. Samples were cut with a cryotome (12 $\mu \mathrm{m}$ sections,CM1900, Leica Microsystems). In Periodic acid-Schiff (PAS) stained sections, capillaries and fibers were counted manually within two $0.25 \mathrm{~mm}^{2}$ squares at $10 \mathrm{x}$ resolution. The ratio of capillaries to muscle fibers was analyzed (15). In ATPase stained sections, the diameter and area of single fibers in a $0.25 \mathrm{~mm}^{2}$ square were edged ( $n=90$ per fiber type) and determined using the NISElements AR 4.0 program (Nikon Instruments Europe, Amsterdam, Netherlands). Type I and type IIb fibers were analyzed in combination (shown as type I fibers).

\section{Serum Analyses}

After decapitation, serum samples were collected to detect levels of calcium, magnesium, phosphorus, alkaline phosphatase (Alp) activity, osteocalcin (OC) and RatLaps. The analyses were conducted at the Department of Clinical Chemistry, University of Goettingen using an Architect 16000 analyzer (Abbott, Wiesbaden, Germany) according to the manufacturer's instructions.

OC was assessed with Rat-MID Osteocalcin EIA (Immunodiagnostic Systems [IDS], Frankfurt am Main, Germany). RatLaps was measured with RatLaps (CTX-I) EIA (IDS).

\section{Statistics}

Statistical analysis was performed with GraphPad Prism 9.0 (GraphPad Software, San Diego, CA, USA) and R 4.0.2 (The R Foundation for Statistical Computing, Vienna, Austria). Normal distribution was assessed with Anderson-Darling test. One-way analysis of variance (ANOVA, $\mathrm{p}<0.05$ ) was applied to detect the impact of the treatments. Differences between the groups were estimated by Tukey's post hoc test with a significance level of 0.05 ( $95 \%$ confidence interval) $(\mathrm{p}<0.05)$. Data are presented as means and standard errors of the mean.

\section{RESULTS}

\section{LOX Inhibition Had No Effect on Body Weight, Uterus Weight, and Food Intake}

While the body weight in the beginning was equally distributed among groups, all OVX groups had higher final weight compared to NON-OVX (Table 1 and Figures 1B, C). Weight of uteri was significantly lower in all OVX groups compared to NON-OVX rats (Table 1 and Figures 1B, C). The treatments with LOX inhibitors affected neither body weight nor uterus weight. The daily food intake was similarly distributed among the groups at the respective week (Figure 1C).

\section{Combination Therapy Increased Serum OC Levels}

The serum electrolytes calcium, magnesium and phosphorus did not differ significantly between the experimental groups (Table 1), whereas Alp was higher in OVX compared to NON-OVX group. RatLaps showed no differences, while OC was upregulated in combination therapy compared to NONOVX control group, indicating a higher bone formation rate. 
TABLE 1 | Body weight, uterus weight, serum analyses, biomechanical and ashing analyses of bone.

\begin{tabular}{|c|c|c|c|c|c|c|c|c|c|c|}
\hline & \multicolumn{2}{|c|}{ NON-OVX } & \multicolumn{2}{|c|}{ ovx } & \multicolumn{2}{|c|}{ Baicalein } & \multicolumn{2}{|c|}{ Zileuton } & \multicolumn{2}{|c|}{ Baicalein+Zileuton } \\
\hline & Mean & SEM & Mean & SEM & Mean & SEM & Mean & SEM & Mean & SEM \\
\hline Weight & $n=8$ & & $\mathrm{n}=10$ & & $n=11$ & & $n=11$ & & $n=10$ & \\
\hline Body weight beginning (g) & 300 & 4.0 & 310 & 4.5 & 306 & 3.3 & 300 & 4.6 & 302 & 4.2 \\
\hline Body weight end $(\mathrm{g})$ & $368^{b-e}$ & 7.5 & 467 & 11.8 & 467 & 7.0 & 451 & 7.9 & 458 & 15.3 \\
\hline Uterus weight $(\mathrm{g})$ & $0.57^{\mathrm{b}-\mathrm{e}}$ & 0.04 & 0.10 & 0.01 & 0.11 & 0.01 & 0.09 & 0.01 & 0.09 & 0.01 \\
\hline Biomechanics - L4 & $\mathrm{n}=7$ & & $\mathrm{n}=9$ & & $\mathrm{n}=9$ & & $\mathrm{n}=11$ & & $\mathrm{n}=10$ & \\
\hline Stiffness [N/mm] & 351 & 28.2 & 298 & 22.5 & 282 & 18.2 & 339 & 16.6 & 327 & 19.7 \\
\hline$F \max [\mathrm{N}]$ & $326^{b-e}$ & 23.4 & 228 & 15.4 & 240 & 20.6 & 253 & 13.8 & 238 & 11.6 \\
\hline Femur & $\mathrm{n}=8$ & & $n=9$ & & $n=11$ & & $\mathrm{n}=11$ & & $n=10$ & \\
\hline Stiffness [N/mm] & 387 & 30.3 & 334 & 19.1 & 324 & 25.8 & 343 & 24.5 & 299 & 19.9 \\
\hline $\mathrm{Fmax}[\mathrm{N}]$ & $194^{\mathrm{b}, \mathrm{e}}$ & 9.6 & 146 & 7.3 & 163 & 9.0 & 163 & 8.2 & 147 & 10.8 \\
\hline Tibia & $\mathrm{n}=7$ & & $\mathrm{n}=10$ & & $\mathrm{n}=11$ & & $\mathrm{n}=11$ & & $n=9$ & \\
\hline Stiffness [N/mm] & 80 & 14.81 & 78 & 15.16 & 73 & 8.77 & 70 & 11.81 & 90 & 18.20 \\
\hline$F \max [\mathrm{N}]$ & 48 & 13.40 & 42 & 7.00 & 58 & 10.19 & 49 & 5.89 & 62 & 7.82 \\
\hline Serum analysis & $n=8$ & & $\mathrm{n}=10$ & & $n=11$ & & $\mathrm{n}=11$ & & $n=10$ & \\
\hline Calcium [mmol/l] & 2.01 & 0.05 & 2.09 & 0.02 & 1.99 & 0.05 & 2.12 & 0.06 & 2.09 & 0.04 \\
\hline Magnesium [mmol/l & 0.70 & 0.02 & 0.73 & 0.02 & 0.70 & 0.02 & 0.75 & 0.03 & 0.73 & 0.01 \\
\hline Phosphorus [mmol/l] & 1.76 & 0.04 & 1.90 & 0.06 & 1.74 & 0.07 & 1.89 & 0.07 & 1.96 & 0.07 \\
\hline Alkaline phosphatase (Alp; U/I) & 114 & 7.5 & $189^{\mathrm{a}}$ & 15.1 & 154 & 11.8 & 176 & 22.7 & 157 & 13.7 \\
\hline Osteocalcin (OC; ng/ml) & 116 & 5.0 & 147 & 11.5 & 177 & 18.0 & 170 & 19.3 & $211^{\mathrm{a}}$ & 31.4 \\
\hline RatLaps (ng/ml) & 5.47 & 1.22 & 9.48 & 0.91 & 9.69 & 1.64 & 9.70 & 1.84 & 10.67 & 1.77 \\
\hline Ashing - L4 & $\mathrm{n}=8$ & & $\mathrm{n}=10$ & & $\mathrm{n}=11$ & & $\mathrm{n}=11$ & & $\mathrm{n}=10$ & \\
\hline$\%$ organic content & $67^{\mathrm{c}, \mathrm{d}}$ & 0.9 & 70 & 0.8 & 71 & 0.8 & 71 & 0.6 & 70 & 0.9 \\
\hline$\%$ anorganic content & $33^{c, d}$ & 0.9 & 30 & 0.8 & 29 & 0.8 & 29 & 0.6 & 30 & 0.9 \\
\hline Femur & $\mathrm{n}=8$ & & $\mathrm{n}=10$ & & $n=11$ & & $\mathrm{n}=11$ & & $n=10$ & \\
\hline$\%$ organic content & $55^{\mathrm{b}-\mathrm{e}}$ & 0.7 & 59 & 0.8 & 58 & 0.8 & 59 & 0.7 & 61 & 1.3 \\
\hline$\%$ anorganic content & $45^{\mathrm{b}-\mathrm{e}}$ & 0.7 & 41 & 0.8 & 42 & 0.8 & 41 & 0.7 & 39 & 1.3 \\
\hline
\end{tabular}

${ }^{a}$ Differs from NON-OVX. ${ }^{b}$ Differs from OVX. ${ }^{c}$ Differs from Baicalein. ${ }^{d}$ Differs from Zileuton. ${ }^{e}$ Differs from Baicalein+Zileuton $(p<0.05)$.

The combination therapy increased serum OC levels but did not affect other serum parameters (Table 1).

\section{The Osteoporotic Phenotype Overweighs the Effect of LOX Inhibitors In In Vivo pQCT and Biomechanical Measurements}

At both time points, before osteotomy ( 8 weeks after OVX) and at the end of the experiment, CSA was significantly higher in all OVX rats compared to NON-OVX irrespective of the treatments (Table 2). BMD of L4 was significantly lower in Baicalein- and Zileuton-treated groups after 8 weeks compared to NON-OVX (Table 2), whereas, at the end, all OVX groups showed lower BMD than NON-OVX rats (Table 2). The SSI was significantly higher in Zileuton compared to the OVX group (Table 2), while in other 3 OVX groups SSI was lower than in NON-OVX group at week 8 after OVX. At the end of the study, only the Baicalein group did not differ from NON-OVX, whereas SSI in the other OVX groups was significantly lower than in NON-OVX (Table 2).

The biomechanical analysis showed a reduction of Fmax in all OVX groups in vertebra and femur, but not of Fmax or stiffness in tibia, where no differences occurred (Table 1).

\section{Zileuton Ameliorated Trabecular Bone in L4, While Zileuton and Combined Therapies Deteriorated Trabecular Properties in the Femur}

In $\mathrm{L} 4$, trabecular $\mathrm{BMD}$, total $\mathrm{BMD}$ and $\mathrm{BV} / \mathrm{TV}$ were reduced in all OVX groups irrespectively of the treatment (Table 3). In the femur, BV/TV was reduced in all OVX groups compared with the NON-OVX group (Table 3), whereas trabecular BMD was impaired in Baicalein and Baicalein+Zileuton groups and total BMD in OVX and Zileuton groups (Table 3). Summarizing, the micro-CT 3D analysis demonstrated a consistent effect of the ovariectomy in all treatment groups.

Micro-CT 2D analysis revealed an improvement in L4 Tb.Dn, N.Nd and Tb.Th as well as Tb.Ar in Zileuton group compared to the OVX group in which an overall reduction of these parameters were observed compared to NON-OVX (Table 3). The cortical density was impaired in Zileuton and combination therapy in L4 (Table 3). In the femur, Zileuton and combination therapy led to reduced Tb.Dn and N.Nd compared to NONOVX and OVX groups (Table 3), while the combination therapy significantly reduced Tb.Th, Tb.Ar and Ct.Dn (Table 3).

Taken together, sole treatment with Baicalein, did not change trabecular or cortical parameters compared to OVX (Table 3). The ovariectomy-induced negative effect on trabecular parameters could be rescued by Zileuton only therapy in L4, but in the femur the effect was contrary. The combination therapy had no effect on bone in L4, while in the femur, the diminishing effect of Zileuton remained after the addition of Baicalein.

\section{Organic Content Is Raised by Baicalein and Zileuton Therapies in L4}

The organic content of L4 was significantly higher and, correspondingly, the anorganic content lower in Baicalein- and 
TABLE 2 | L4, in Vivo PQCT.

\begin{tabular}{|c|c|c|c|c|c|c|c|c|c|c|}
\hline & \multicolumn{2}{|c|}{ NON-OVX $(n=5)$} & \multicolumn{2}{|c|}{ OVX $(n=5)$} & \multicolumn{2}{|c|}{ Baicalein $(n=5)$} & \multicolumn{2}{|c|}{ Zileuton $(n=5)$} & \multicolumn{2}{|c|}{ Baicalein+Zileuton $(n=5)$} \\
\hline & Mean & SEM & Mean & SEM & Mean & SEM & Mean & SEM & Mean & SEM \\
\hline \multicolumn{11}{|l|}{$\operatorname{CSA}\left(\mathrm{mm}^{2}\right)$} \\
\hline 8 w after OVx & $1886^{\mathrm{b}-\mathrm{e}}$ & 39.73 & 2306 & 39.91 & 2381 & 78.71 & 2380 & 32.38 & 2321 & 50.23 \\
\hline $13 w$ after OVX & $1866^{b-e}$ & 25.63 & 2284 & 58.66 & 2256 & 22.47 & 2438 & 41.57 & 2424 & 47.05 \\
\hline \multicolumn{11}{|c|}{ BMD L4 (mg/cm³) } \\
\hline $8 w$ after OVX & $585.0^{\mathrm{c}, \mathrm{d}}$ & 6.9 & 551.2 & 10.3 & 531.5 & 9.1 & 536.1 & 12.4 & 547.5 & 9.1 \\
\hline $13 w$ after OVX & $554.8^{\mathrm{b}-\mathrm{e}}$ & 7.8 & 511.9 & 7.8 & 505.2 & 5.6 & 502.2 & 7.7 & 506.5 & 8.4 \\
\hline \multicolumn{11}{|l|}{ SSI L4 } \\
\hline $8 w$ after OVX & $19.91^{b, c, e}$ & 0.75 & $15.59^{d}$ & 0.32 & 15.84 & 0.38 & 18.43 & 0.82 & 16.79 & 0.58 \\
\hline 13w after OVX & $18.21^{\mathrm{b}, \mathrm{d}, \mathrm{e}}$ & 0.73 & 14.94 & 0.74 & 16.91 & 1.06 & 14.71 & 0.47 & 14.61 & 0.55 \\
\hline
\end{tabular}

${ }^{b}$ Differs from OVX. ${ }^{c}$ Differs from Baicalein. ${ }^{d}$ Differs from Zileuton. ${ }^{e}$ Differs from Baicalein+Zileuton $(p<0.05)$.

Zileuton only treated groups than in the NON-OVX group (Table 1). Organic content of femora was detected to be lower in the NON-OVX group than in all other groups, while the anorganic weight showed the opposite results (Table 1).

\section{Baicalein Accelerated Mid-Stage Callus Formation, While Zileuton and Combination Therapies Improved Cortical Parameters in the Tibia}

Analyses of fluorochrome stained sections of the tibia (Figure 2E) revealed no differences in the time of the first osseous bridging of osteotomized bone ends (mean: $24 \pm 1$ day after osteotomy). The late ventral callus area (28-33d after osteotomy, TC staining) was significantly larger in NON-OVX animals compared to all other treatment groups (Figure 2A). Moreover, the late dorsal callus area was larger in NON-OVX compared to Zileuton and combination therapy groups (Figure 2B). Baicalein led to a larger midtime area (20-27d after osteotomy, AC staining) of dorsal callus area compared to the OVX group. Midtime endosteal callus area (AC, Figure 2C) and total callus area (Figure 2D) were larger in the Baicalein group than in the combination therapy group. The late total callus area (TC staining) in the NON-OVX group exceeded the OVX group (Figure 2D).

TABLE 3 | micro-CT of L4, femora and tibiae, 3D and 2D analyses.

\begin{tabular}{|c|c|c|c|c|c|c|c|c|c|c|}
\hline & \multicolumn{2}{|c|}{ NON-OVX } & \multicolumn{2}{|c|}{ ovx } & \multicolumn{2}{|c|}{ Baicalein } & \multicolumn{2}{|c|}{ Zileuton } & \multicolumn{2}{|c|}{ Baicalein+Zileuton } \\
\hline & Mean & SEM & Mean & SEM & Mean & SEM & Mean & SEM & Mean & SEM \\
\hline L4, 3D & $n=8$ & & $n=10$ & & $n=10$ & & $\mathrm{n}=11$ & & $n=10$ & \\
\hline Tb. BMD $\left(\mathrm{g} / \mathrm{cm}^{3}\right)$ & $0.634^{\mathrm{b}-\mathrm{e}}$ & 0.004 & 0.611 & 0.002 & 0.609 & 0.003 & 0.614 & 0.002 & 0.607 & 0.001 \\
\hline Total BMD (g/cm³) & $0.606^{\mathrm{b}-\mathrm{e}}$ & 0.013 & 0.509 & 0.011 & 0.496 & 0.013 & 0.519 & 0.011 & 0.481 & 0.007 \\
\hline $\mathrm{BV} / \mathrm{TV}(\%)$ & $70.67^{\mathrm{b}-\mathrm{e}}$ & 1.02 & 59.40 & 1.09 & 59.15 & 1.24 & 62.02 & 1.11 & 58.27 & 0.85 \\
\hline L4,2D & $\mathrm{n}=8$ & & $n=10$ & & $n=10$ & & $\mathrm{n}=11$ & & $\mathrm{n}=10$ & \\
\hline Tb.Dn (\%) & $44.18^{\mathrm{b}-\mathrm{e}}$ & 1.126 & 26.37 & 0.6563 & 26.66 & 0.8595 & $31.44^{\mathrm{b}, \mathrm{c}, \mathrm{e}}$ & 0.9328 & 27.86 & 0.7008 \\
\hline N.Nd (n) & $40.63^{b-e}$ & 1.508 & 31.53 & 0.8033 & 29.70 & 1.387 & $34.79^{b, c}$ & 0.9342 & 32.52 & 0.9605 \\
\hline Tb.Th (mm) & $0.25^{\mathrm{b}-\mathrm{e}}$ & 0.01 & $0.13^{d}$ & 0.01 & 0.14 & 0.01 & 0.17 & 0.01 & 0.15 & 0.01 \\
\hline Tb.Ar $\left(\mathrm{mm}^{2}\right)$ & $7.45^{\mathrm{b}-\mathrm{e}}$ & 0.23 & 4.84 & 0.11 & 4.80 & 0.17 & $5.85^{\mathrm{b}, \mathrm{c}, \mathrm{e}}$ & 0.21 & 5.12 & 0.13 \\
\hline Ct.Dn (\%) & $94.43^{\mathrm{d}, \mathrm{e}}$ & 0.77 & 90.99 & 0.49 & 91.62 & 0.60 & 87.48 & 2.70 & 86.86 & 0.65 \\
\hline Femur, 3D & $\mathrm{n}=8$ & & $n=10$ & & $n=11$ & & $\mathrm{n}=11$ & & $n=10$ & \\
\hline Tb. BMD $\left(\mathrm{g} / \mathrm{cm}^{3}\right)$ & $0.871^{\mathrm{c}, \mathrm{e}}$ & 0.006 & 0.854 & 0.003 & 0.848 & 0.007 & 0.852 & 0.004 & 0.849 & 0.004 \\
\hline Total BMD (g/cm³) & $0.822^{b, d}$ & 0.021 & 0.663 & 0.017 & 0.726 & 0.029 & 0.695 & 0.026 & 0.690 & 0.014 \\
\hline BV/TV (\%) & $76.46^{\mathrm{b}-\mathrm{e}}$ & 1.68 & 60.65 & 2.46 & 65.27 & 2.49 & 65.53 & 2.38 & 64.09 & 1.22 \\
\hline Femur, 2D & $\mathrm{n}=8$ & & $\mathrm{n}=10$ & & $n=11$ & & $\mathrm{n}=11$ & & $\mathrm{n}=10$ & \\
\hline Tb.Dn (\%) & $49.18^{\mathrm{b}-\mathrm{e}}$ & 2.323 & $32.30^{\mathrm{d}, \mathrm{e}}$ & 1.995 & $29.96^{\mathrm{e}}$ & 1.616 & 23.79 & 1.372 & 21.63 & 1.177 \\
\hline N.Nd (n) & $73.54^{\mathrm{b}-\mathrm{e}}$ & 2.44 & $38.40^{\mathrm{d}, \mathrm{e}}$ & 2.71 & 32.87 & 2.32 & 30.18 & 1.55 & $20.52^{\mathrm{c}, \mathrm{d}}$ & 0.92 \\
\hline Tb.Th $(\mu \mathrm{m})$ & $121^{\mathrm{b}-\mathrm{e}}$ & 5.8 & 89 & 2.4 & 86 & 2.4 & 81 & 1.5 & $74^{\mathrm{b}, \mathrm{c}}$ & 1.4 \\
\hline Tb.Ar $\left(\mathrm{mm}^{2}\right)$ & $5.75^{\mathrm{b}-\mathrm{e}}$ & 0.18 & $3.67^{\mathrm{c}-\mathrm{e}}$ & 0.20 & 2.97 & 0.15 & 2.95 & 0.11 & $2.27^{c, d}$ & 0.09 \\
\hline Ct.Dn (\%) & 97.49 & 0.24 & 97.20 & 0.26 & 96.51 & 0.43 & 96.71 & 0.30 & $95.19^{a, b, d}$ & 0.52 \\
\hline Tibia, 3D & $\mathrm{n}=7$ & & $n=10$ & & $n=10$ & & $\mathrm{n}=10$ & & $n=10$ & \\
\hline Ct. BMD (g/cm³) & $1.116^{\mathrm{d}, \mathrm{e}}$ & 0.034 & $1.083^{\mathrm{d}, \mathrm{e}}$ & 0.030 & 1.158 & 0.025 & 1.210 & 0.008 & 1.214 & 0.007 \\
\hline Ct.V $\left(\mathrm{mm}^{3}\right)$ & 20.40 & 5.158 & $14.43^{\mathrm{d}, \mathrm{e}}$ & 4.243 & 27.52 & 4.168 & 34.84 & 1.550 & 29.03 & 1.680 \\
\hline Total bone BMD (g/cm³) & 0.585 & 0.025 & 0.519 & 0.024 & 0.522 & 0.018 & 0.532 & 0.021 & 0.496 & 0.019 \\
\hline Cl. BMD $\left(\mathrm{g} / \mathrm{cm}^{3}\right)$ & $0.392^{\mathrm{b}-\mathrm{e}}$ & 0.012 & 0.326 & 0.017 & 0.333 & 0.005 & 0.331 & 0.007 & 0.327 & 0.012 \\
\hline Osseous Cl.V/Total Cl.V BV/TV & $66.30^{\mathrm{b}, \mathrm{e}}$ & 2.19 & 57.67 & 2.534 & 59.18 & 1.09 & 59.16 & 1.50 & 55.98 & 1.77 \\
\hline
\end{tabular}

${ }^{a}$ Differs from NON-OVX. ${ }^{b}$ Differs from OVX. ${ }^{c}$ Differs from Baicalein. ${ }^{d}$ Differs from Zileuton. ${ }^{e}$ Differs from Baicalein+Zileuton $(p<0.05)$. 


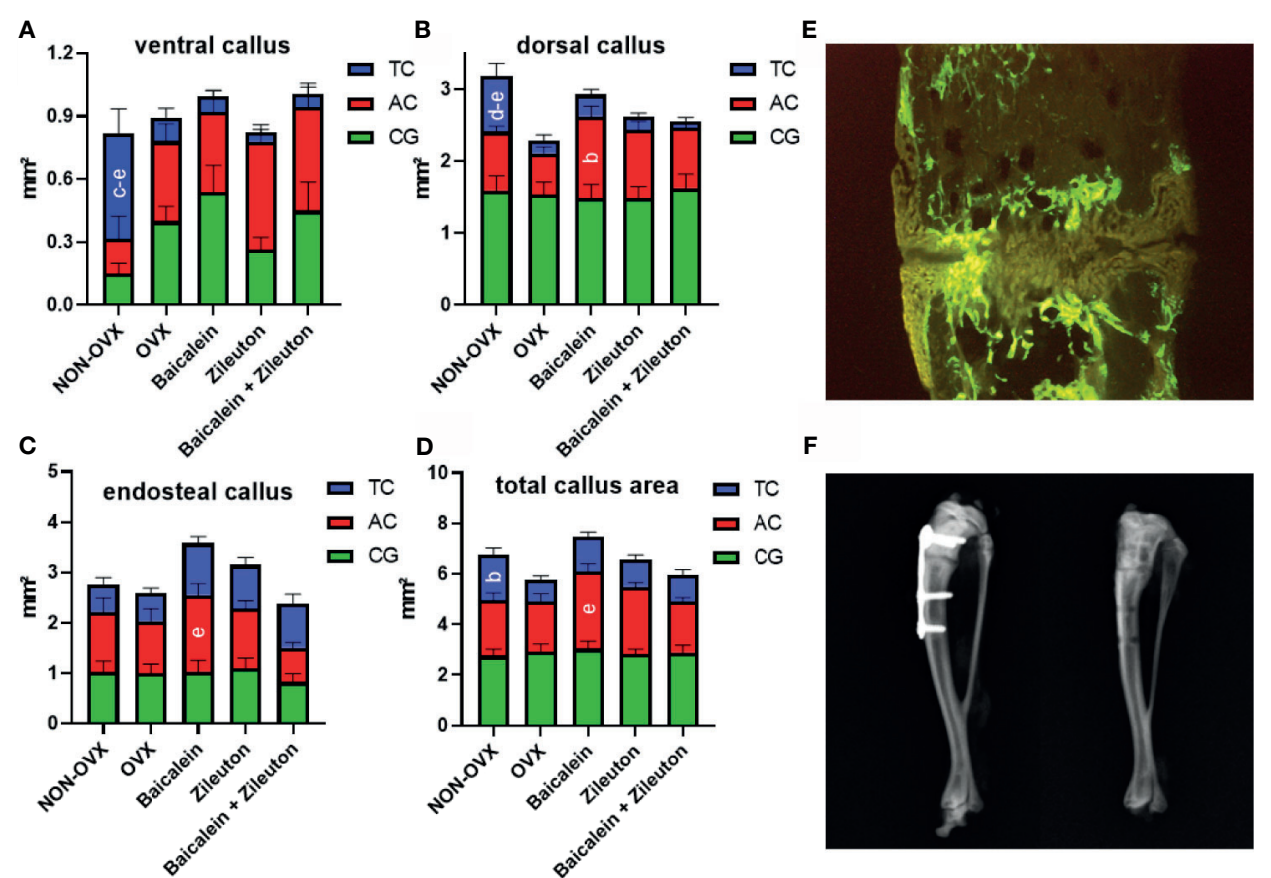

FIGURE 2 | Tibia healing analysis. Ventral callus area (A), dorsal callus area (B), endosteal callus area (C) and total callus area (D) stained with fluorochromes $\mathrm{XO}+\mathrm{CG}$ (0-19d after osteotomy), AC (20-27d after osteotomy) and TC (28-33d after osteotomy) and measured on histological sections (E). X-rays prove the correct positioning of the plate after tibial osteotomy and healing is confirmed after plate removal (F). [NON-OVX: $n=8 ;$ OVX: $n=10 ;$ Baicalein: $n=9 ;$ Zileuton: $n=11$; Baicalein + Zileuton: $n=11$ (3 histological sections per animal)]. ${ }^{b}$ Differs from OVX. 'Differs from Baicalein. ${ }^{d}$ Differs from Zileuton. ${ }^{e}$ Differs from Baicalein+Zileuton ( $\left.p<0.05\right)$.

Micro-CT 3D analysis showed enhanced Ct. BMD and Ct.V in the tibia in both Zileuton and combination therapy groups (Table 3), while total Cl. BMD was reduced in all OVX groups (Table 3). The osseous callus volume fraction, however, was lowest in the combination therapy group (Table 3), while total bone BMD did not differ among the groups (Table 3 ).

Summarizing, Baicalein treatment resulted in enhanced endosteal and total callus area compared to combination therapy and increased dorsal callus area compared to the OVX group. The cortical parameters in the tibia were enhanced by Zileuton and combination therapy compared to OVX.

\section{Combination Therapy Enhanced Capillary Ratio in M. Gastrocnemius}

In Musculus gastrocnemius, soleus and longissimus neither diameter nor area of muscle fibers differed significantly among treatment groups (Table 4). The combination therapy in M. gastrocnemius led to a higher capillary ratio compared to NONOVX and OVX groups, which was not noticeable in $\mathrm{M}$. longissimus and M. soleus (Table 4).

\section{DISCUSSION}

Osteoporosis as a systemic disease is manifested on both cortical and trabecular bone. The detection of osteoporosis as well as the therapeutic success are closely connected to the structural changes in spine and proximal femur $(28,29)$. Due to an aging society, an increasing socioeconomic relevance is attributed to this "silent disease" and the need for new therapeutic approaches is evident $(5,30,31)$. Recently, we have reported promising results of sole Baicalein and Zileuton treatments on osteoporotic bone tissue $(13,14,16)$, though some undesirable side-effects resulted from subcutaneous administration of the former. We subsequently decided on an oral administration of both LOX inhibitors. A oral administration of Baicalein in a dose of $10 \mathrm{mg} / \mathrm{kg}$ per day exhibited inhibitory effects in a xenograft experiment in mice (32), while in a rat experiment, an oral dose of 10 and $20 \mathrm{mg} / \mathrm{kg}$ Baicalein led to a stable concentration of its active metabolite, Baicalin, in the rat plasma (17).

In the present study, we investigated the effect of a combined treatment of Baicalein and Zileuton as well as single treatments of these substances on lumbar vertebral body and femur, and on bone healing in the osteotomized tibia as well as on the skeletal muscle in an ovariectomized rat model of postmenopausal osteoporosis $(13,22)$. Effects of the LOX inhibitors on body weight, uterus weight or food intake were ruled out since the treatments did not lead to differences in these compared to the OVX group. We did not detect effects of these substances on the whole body composition in our previous studies either (13, 16, 19). 
TABLE 4 | Histological analyses of M. Gastrocnemius (MG), M. Longissimus (ML) and M. Soleus (MS).

\begin{tabular}{|c|c|c|c|c|c|c|c|c|c|c|}
\hline & \multicolumn{2}{|c|}{ NON-OVX $(n=8)$} & \multicolumn{2}{|c|}{ OVX $(n=10)$} & \multicolumn{2}{|c|}{ Baicalein $(n=10)$} & \multicolumn{2}{|c|}{ Zileuton $(n=11)$} & \multicolumn{2}{|c|}{ Baicalein+Zileuton $(n=10)$} \\
\hline & Mean & SEM & Mean & SEM & Mean & SEM & Mean & SEM & Mean & SEM \\
\hline \multicolumn{11}{|l|}{ MG, type I } \\
\hline Diameter $(\mu \mathrm{m})$ & 48.91 & 2.76 & 50.91 & 1.79 & 51.42 & 1.22 & 50.25 & 1.75 & 50.01 & 1.72 \\
\hline Area $\left(\mu \mathrm{m}^{2}\right)$ & 1939 & 222 & 2094 & 135 & 2094 & 101 & 2048 & 148 & 2034 & 135 \\
\hline \multicolumn{11}{|l|}{ MG, type Ila } \\
\hline Diameter $(\mu \mathrm{m})$ & 71.84 & 2.54 & 76.42 & 2.25 & 77.17 & 2.38 & 73.29 & 1.28 & 72.78 & 2.44 \\
\hline Area $\left(\mu m^{2}\right)$ & 4153 & 309 & 4699 & 277 & 4790 & 289 & 4285 & 154 & 4098 & 319 \\
\hline \multicolumn{11}{|c|}{ MG, capillarization } \\
\hline Capillary/Fiber & 1.22 & 0.05 & 1.27 & 0.04 & 1.45 & 0.075 & 1.39 & 0.077 & $1.67^{a, b}$ & 0.10 \\
\hline \multicolumn{11}{|l|}{ ML, type I } \\
\hline Diameter $(\mu \mathrm{m})$ & 56.64 & 1.83 & 56.84 & 1.16 & 54.46 & 1.94 & 57.16 & 1.25 & 54.55 & 2.21 \\
\hline Area $\left(\mu \mathrm{m}^{2}\right)$ & 2619 & 168 & 2606 & 109 & 2390 & 176 & 2610 & 117 & 2372 & 183 \\
\hline \multicolumn{11}{|l|}{ ML, type Ila } \\
\hline Diameter $(\mu \mathrm{m})$ & 82.81 & 0.84 & 88.71 & 1.86 & 81.90 & 3.18 & 84.99 & 1.62 & 83.32 & 3.09 \\
\hline Area $\left(\mu m^{2}\right)$ & 5442 & 115 & 6248 & 317 & 5387 & 412 & 5725 & 230 & 5551 & 407 \\
\hline \multicolumn{11}{|c|}{ ML, capillarization } \\
\hline Capillary/Fiber & 1.15 & 0.09 & 1.38 & 0.07 & 1.46 & 0.08 & 1.42 & 0.07 & 1.38 & 0.08 \\
\hline \multicolumn{11}{|l|}{ MS, type I } \\
\hline Diameter $(\mu \mathrm{m})$ & 68.39 & 1.33 & 66.93 & 2.48 & 67.50 & 1.34 & 67.73 & 1.50 & 66.90 & 0.73 \\
\hline Area $\left(\mu \mathrm{m}^{2}\right)$ & 3733 & 145.9 & 3604 & 287.5 & 3628 & 146.9 & 3665 & 168.5 & 3554 & 76.18 \\
\hline \multicolumn{11}{|c|}{ MS, capillarization } \\
\hline Capillary/Fiber & 1.57 & 0.06 & 1.81 & 0.08 & 1.57 & 0.06 & 1.59 & 0.07 & 1.71 & 0.11 \\
\hline
\end{tabular}

${ }^{a}$ Differs from NON-OVX. ${ }^{b}$ Differs from OVX.

Previously, we detected favorable effects on the capillarization of muscle after subcutaneous injection of Baicalein (15) and oral administration of Zileuton (unpublished data) for up to 4-5 weeks. In the present study, the effect was less pronounced. Solely the combination therapy increased capillarization in $\mathrm{M}$. gastrocnemius. One reason might be that the muscular effect is short-lasting, leading to detectable effects after short-term treatments [as in our previous study (15)], but diminishing due to adaption after 13 weeks. Another possible reason might root in the time of administration. In this study, the LOX inhibitors were applied as preventive treatments, immediately after OVX, whereas previously they were applied 8 weeks after OVX when changes in the musculoskeletal system due to hormone deficiency had already manifested (33).

In our in vivo $\mathrm{pQCT}$, no effect of LOX inhibitors was observed on CSA. The combination therapy did not change SSI of L4, whereas Zileuton-treatment showed higher SSI than that in the OVX group after an 8 week treatment, which is comparable to our previous 5 week treatment (13). The oral route of administration for Baicalein was demonstrated to be safe, however it showed no effect on BMD and SSI in the in vivo pQCT analyses. The micro-CT 3D analysis revealed no effect of either therapy on bone parameters, whereas the $2 \mathrm{D}$ analysis illustrated a beneficial effect of Zileuton on trabecular bone parameters in the lumbar spine. Thus, the previously reported trabecular accentuated effects of Zileuton on the vertebra (13) could again be confirmed after the longer treatment regimen for 13 weeks in this study. In contrast to these findings in L4, the trabecular bone was impaired in femur after Zileuton and combination treatments. Furthermore, the cortical bone lost its density after these treatments in L4, too. After a short-term application, the Zileuton treatment caused an increase of bone volume in the femur, whereas femoral density was reduced (14). An oral Baicalein treatment did not change parameters measured by micro-CT in the present study. Hence, we supported our previous findings of Zileuton being favorable in trabecular bone in spine and unfavorable in femur (13), while Baicalein did not show any effect on bone and did not reduce the negative effect of Zileuton in the combination therapy in femur.

Proximal femur fractures, which occur commonly in the aged people, constitute a devastating diagnosis with 1-year mortality rates of up to $36 \%$, regularly indicating missed anti-osteoporotic therapy (34). The previously reported effects of Baicalein were sparse (16), while cortical volume in the distal femur was significantly ameliorated after Zileuton-therapy in a dose of $10 \mathrm{mg} / \mathrm{kg} \mathrm{BW}$ (14). Three-dimensional analysis of femoral head confirmed weak effects of Baicalein. The advantage of Zileuton on cortical volume was not consistent after prolonged therapy in our study. The combination therapy, however, reduced Tb.Th and Ct.Dn substantially. All of these findings put the combination therapy in an unfavorable light.

In our bone healing analysis, a favorable effect of sole Baicalein therapy was detected in the dorsal callus area, mostly in the midterm healing period. Indeed, the main effect of both Baicalein and Zileuton as their combination therapy on ventral and dorsal callus formation was observed within 20 and 33 days after osteotomy. In a former study, we could detect an impairment of early callus formation after subcutaneous Baicalein administration (16). The oral treatment with either Baicalein, Zileuton or both in the current study showed a reduction in these groups in the late ventral callus formation, whereas the cortical parameters were improved after Zileuton 
und combination therapies at the osteotomy site. Contrary to our descriptions of a partial deterioration of fracture healing via pharmacological lipoxygenase-inhibition, the positive effect of a lipoxygenase knockout on fracture healing was demonstrated in a $5-L O(\mathrm{KO})$ mouse model by Manigrasso et al. (29). In their knockout model, the authors observed enhanced healing with substantially better mechanical properties of the newly formed bone compared to wild type mice (35).

However, the exact underlying molecular mechanism of Baicalein's mode of action is not understood in detail. Several key enzymes like 5-, 12- and 15-LOX are inhibited next to cyclooxygenases (COX 1 and 2), resulting in reduced synthesis of prostaglandins $(36,37)$. Intriguingly, both Zileuton and Baicalein have been demonstrated to inhibit stress-mediated 5LOX metabolite (cysteinyl leukotrienes) production, which may be a reason for the lack of synergistic effects in our study (36). Besides, Baicalein was demonstrated to inhibit parathormone (PTH)-induced DNA synthesis and cytokines like IL-6 and TNF$\alpha(38,39)$. Further stimulating osteoblast differentiation, the induction of $N F-\kappa B$ and nuclear factor of activated T-cells, cytoplasmic 1 (NFATc1) as well as the induction of osteoclast apoptosis have been described, resulting in an overall osteogenic effect $(40,41)$. In our previous study, we speculated that Baicalein had an antioxidative effect next to osteoclast apoptosis (via Wnt/ $\beta$-catenin pathway) and radical-scavenging effect (16). The main effect of Zileuton is created via 5-LOX inhibition. While in vivo data show modulation of cytokine release and reduced osteoclast differentiation (42), additionally giving rise to osteoanabolic reactions, our own analysis showed an upregulation of osteocalcin and Alp in the serum $(14,42)$. Despite the bone healing analyses showed no sweeping effects of Zileuton and combination therapy on callus density, callus area and cortical bone (Ct.BMD and Ct.V), the healed cortical tibia (Ct.BMD, Ct.V) was favorably affected by these treatments. A connecting role may be attributed to the raised serum levels of osteocalcin which we measured in combination therapy. These have been similarly described after 5-LOX inhibition elsewhere $(16,43)$.

\section{CONCLUSION}

Summarizing, the spinal trabecular bone was improved by Zileuton, but not by combination therapy, which is even in disfavor for Tb.BMD, BV/TV and Ct.Dn. The analysis of the clinically crucial proximal femur could not show the expected ameliorative influence of LOX inhibitors, but instead clarified plain negative consequences of combined therapy of Baicalein and Zileuton on cortical and trabecular bone. The oral administration of Baicalein - despite a longer therapy period did not lead to the same beneficial cortical effects as described earlier (16). Though the dynamic bone healing process was favorably affected by all LOX inhibitor treatments, their overall effect on bone healing was minor. Furthermore, inhibition of LOX did not have a profound benefit on the skeletal muscle.

Taken together, our data showed no perspective of both lipoxygenase inhibitors neither alone or in combination for the musculoskeletal system in estrogen deficient rats and particularly not as a preventive treatment of osteoporosis.

\section{Limitations}

The oral administration of Baicalein instead of the previously reported subcutaneous administration - due to reported negative side effects - might have affected the results (15). The lack of assessment of metabolic parameters in our rat model yet limits the mechanistic insights of Baicalein and Zileuton action.

\section{DATA AVAILABILITY STATEMENT}

The raw data supporting the conclusions of this article will be made available by the authors, without undue reservation.

\section{ETHICS STATEMENT}

The animal study was reviewed and approved by the the local district government (Oldenburg, Germany) and in compliance with the ethical standards of animal care.

\section{AUTHOR CONTRIBUTIONS}

MK and SS designed the study. FH, MF, IM, and ST performed all experimental procedures. Data analysis was carried out by DS and MK. DS wrote the manuscript with the help of MK. DS, FH, MF, IM, ST, PR, SS, and MK critically revised it for important intellectual content. All authors contributed to the article and approved the submitted version.

\section{FUNDING}

DS was funded by the Deutsche Forschungsgemeinschaft (DFG, German Research Foundation) - 413501650. We thank the Elsbeth Bonhoff Stiftung for financial support (Grant N114).

\section{ACKNOWLEDGMENTS}

The authors thank R. Castro-Machguth,A. Witt and R. Wigger for technical support.

\section{SUPPLEMENTARY MATERIAL}

The Supplementary Material for this article can be found online at: https://www.frontiersin.org/articles/10.3389/fendo.2021.706504/ full\#supplementary-material 


\section{REFERENCES}

1. Reginster J-Y, Burlet N. Osteoporosis: A Still Increasing Prevalence. Bone (2006) 38:S4-9. doi: 10.1016/j.bone.2005.11.024

2. Watts NB, Bilezikian JP, Camacho PM, Greenspan SL, Harris ST, Hodgson SF, et al. American Association of Clinical Endocrinologists Medical Guidelines for Clinical Practice for the Diagnosis and Treatment of Postmenopausal Osteoporosis. Endocr Pract (2010) 16(Suppl 3):1-37. doi: 10.4158/EP.16.S3.1

3. Hansen D, Bazell C, Pelizzari P, Pyenson B. Medicare Cost of Osteoporotic Fractures: The Clinical and Cost Burden of an Important Consequence of Osteoporosis (2020). Available at: https://staticl.squarespace.com/static/ 5c0860aff793924efe2230f3/t/5d76b949deb7e9086ee3d7dd/1568061771769/ Medicare+Cost+of+Osteoporotic+Fractures+20190827.pdf. (Accessed March 08, 2021).

4. Brown C. Osteoporosis: Staying Strong. Nature (2017) 550:S15-7. doi: $10.1038 / 550 S 15 a$

5. Makras P, Delaroudis S, Anastasilakis AD. Novel Therapies for Osteoporosis. Metabolism (2015) 64:1199-214. doi: 10.1016/j.metabol.2015.07.011

6. Cosman F, Crittenden DB, Adachi JD, Binkley N, Czerwinski E, Ferrari S, et al. Romosozumab Treatment in Postmenopausal Women With Osteoporosis. N Engl J Med (2016) 375:1532-43. doi: 10.1056/ NEJMoa1607948

7. Cummings SR, San Martin J, McClung MR, Siris ES, Eastell R, Reid IR, et al. Denosumab for Prevention of Fractures in Postmenopausal Women With Osteoporosis. N Engl J Med (2009) 361:756-65. doi: 10.1056/NEJMoa0809493

8. Kang J-H, Ting Z, Moon M, Sim J-S, Lee J-M, Doh K-E, et al. 5-Lipoxygenase Inhibitors Suppress RANKL-Induced Osteoclast Formation Via NFATc1 Expression. Bioorg Med Chem (2015) 23:7069-78. doi: 10.1016/ j.bmc.2015.09.025

9. Cottrell JA, Keshav V, Mitchell A, O'Connor JP. Local Inhibition of 5Lipoxygenase Enhances Bone Formation in a Rat Model. Bone Joint Res (2013) 2:41-50. doi: 10.1302/2046-3758.22.2000066

10. Kang KA, Zhang R, Piao MJ, Chae S, Kim HS, Park JH, et al. Baicalein Inhibits Oxidative Stress-Induced Cellular Damage Via Antioxidant Effects. Toxicol Ind Health (2012) 28:412-21. doi: 10.1177/0748233711413799

11. Wu Q-Q, Deng W, Xiao Y, Chen J-J, Liu C, Wang J, et al. The 5-Lipoxygenase Inhibitor Zileuton Protects Pressure Overload-Induced Cardiac Remodeling Via Activating PPAR $\alpha$. Oxid Med Cell Longev (2019) 2019:7536803. doi: $10.1155 / 2019 / 7536803$

12. Shi L, Hao Z, Zhang S, Wei M, Lu B, Wang Z, et al. Baicalein and Baicalin Alleviate Acetaminophen-Induced Liver Injury by Activating Nrf2 Antioxidative Pathway: The Involvement of ERK1/2 and PKC. Biochem Pharmacol (2018) 150:9-23. doi: 10.1016/j.bcp.2018.01.026

13. Saul D, Gleitz S, Nguyen HH, Kosinsky RL, Sehmisch S, Hoffmann DB, et al. Effect of the Lipoxygenase-Inhibitors Baicalein and Zileuton on the Vertebra in Ovariectomized Rats. Bone (2017) 101:134-44. doi: 10.1016/j.bone.2017.04.011

14. Saul D, Ninkovic M, Komrakova M, Wolff L, Simka P, Gasimov T, et al. Effect of Zileuton on Osteoporotic Bone and its Healing, Expression of Bone, and Brain Genes in Rats. J Appl Physiol (Bethesda Md 1985) (2018) 124:118-30. doi: 10.1152/japplphysiol.01126.2016

15. Saul D, Kling JH, Kosinsky RL, Hoffmann DB, Komrakova M, Wicke M, et al. Effect of the Lipoxygenase Inhibitor Baicalein on Muscles in Ovariectomized Rats. J Nutr Metab (2016) 2016:3703216. doi: 10.1155/2016/3703216

16. Saul D, Weber M, Zimmermann MH, Kosinsky RL, Hoffmann DB, Menger B, et al. Effect of the Lipoxygenase Inhibitor Baicalein on Bone Tissue and Bone Healing in Ovariectomized Rats. Nutr Metab (2019) 16:4. doi: 10.1186/ s12986-018-0327-2

17. Kim YH, Jeong DW, Kim Y-C, Sohn DH, Park E-S, Lee HS. Pharmacokinetics of Baicalein, Baicalin and Wogonin After Oral Administration of a Standardized Extract of Scutellaria Baicalensis, PF-2405 in Rats. Arch Pharm Res (2007) 30:260-5. doi: 10.1007/BF02977703

18. Kim DH, Hossain MA, Kang YJ, Jang JY, Lee YJ, Im E, et al. Baicalein, an Active Component of Scutellaria Baicalensis Georgi, Induces Apoptosis in Human Colon Cancer Cells and Prevents AOM/DSS-induced Colon Cancer in Mice. Int J Oncol (2013) 43:1652-8. doi: 10.3892/ijo.2013.2086

19. Komrakova M, Fiebig J, Hoffmann DB, Krischek C, Lehmann W, Stuermer KM, et al. The Advantages of Bilateral Osteotomy Over Unilateral Osteotomy for Osteoporotic Bone Healing. Calcif Tissue Int (2018) 103:80-94 doi: 10.1007/s00223-018-0392-6

20. Smolinske SC, Hall AH, Vandenberg SA, Spoerke DG, McBride PV. Toxic Effects of Nonsteroidal Anti-Inflammatory Drugs in Overdose. An Overview of Recent Evidence on Clinical Effects and Dose-Response Relationships. Drug Saf (1990) 5:252-74. doi: 10.2165/00002018-199005040-00003

21. Komrakova M, Hoffmann DB, Nuehnen V, Stueber H, Wassmann M, Wicke M, et al. The Effect of Vibration Treatments Combined With Teriparatide or Strontium Ranelate on Bone Healing and Muscle in Ovariectomized Rats. Calcif Tissue Int (2016) 99:408-22. doi: 10.1007/s00223-016-0156-0

22. Komrakova M, Weidemann A, Dullin C, Ebert J, Tezval M, Stuermer KM et al. The Impact of Strontium Ranelate on Metaphyseal Bone Healing in Ovariectomized Rats. Calcif Tissue Int (2015) 97:391-401. doi: 10.1007/ s00223-015-0019-0

23. Tezval M, Stuermer EK, Sehmisch S, Rack T, Stary A, Stebener M, et al. Improvement of Trochanteric Bone Quality in an Osteoporosis Model After Short-Term Treatment With Parathyroid Hormone: A New Mechanical Test for Trochanteric Region of Rat Femur. Osteoporos Int (2010) 21:251-61. doi: 10.1007/s00198-009-0941-y

24. Sehmisch S, Erren M, Rack T, Tezval M, Seidlova-Wuttke D, Richter J, et al. Short-Term Effects of Parathyroid Hormone on Rat Lumbar Vertebrae. Spine (2009) 34:2014-21. doi: 10.1097/BRS.0b013e3181afe846

25. Parfitt AM, Drezner MK, Glorieux FH, Kanis JA, Malluche H, Meunier PJ, et al. Bone Histomorphometry: Standardization of Nomenclature, Symbols, and Units. Report of the ASBMR Histomorphometry Nomenclature Committee. J Bone Miner Res (1987) 2:595-610. doi: 10.1002/ jbmr.5650020617

26. Bouxsein ML, Boyd SK, Christiansen BA, Guldberg RE, Jepsen KJ, Müller R. Guidelines for Assessment of Bone Microstructure in Rodents Using MicroComputed Tomography. J Bone Miner Res (2010) 25:1468-86. doi: 10.1002/ jbmr.141

27. Komrakova M, Stuermer EK, Sturm A, Schmelz U, Tezval M, Stuermer KM, et al. Efficiency of $48 \mathrm{~h}$ vs. $24 \mathrm{~h}$ Injection of Parathyroid Hormone for Amelioration of Osteopenic Spine Properties in Male Rats. Open Bone J (2012) 4:20-6. doi: 10.2174/1876525401204010020

28. Siris ES, Adler R, Bilezikian J, Bolognese M, Dawson-Hughes B, Favus MJ, et al. The Clinical Diagnosis of Osteoporosis: A Position Statement From the National Bone Health Alliance Working Group. Osteoporos Int (2014) 25:1439-43. doi: 10.1007/s00198-014-2655-z

29. Wright NC, Saag KG, Dawson-Hughes B, Khosla S, Siris ES. The Impact of the New National Bone Health Alliance (NBHA) Diagnostic Criteria on the Prevalence of Osteoporosis in the USA. Osteoporos Int (2017) 28:1225-32. doi: 10.1007/s00198-016-3865-3

30. Rachner TD, Khosla S, Hofbauer LC. Osteoporosis: Now and the Future. Lancet (London England) (2011) 377:1276-87. doi: 10.1016/S0140-6736(10) 62349-5

31. Watts NB, Bilezikian JP. Advances in Target-Specific Therapy for Osteoporosis. J Clin Endocrinol Metab (2014) 99:1149-51. doi: 10.1210/ jc.2014-1065

32. Miocinovic R, McCabe N, Keck RW, Jankun J, Hampton JA, Selman SH. In Vivo and In Vitro Effect of Baicalein on Human Prostate Cancer Cells. Int J Oncol (2005) 26(1):241-6. doi: 10.3892/ijo.26.1.241

33. Yousefzadeh N, Kashfi K, Jeddi S, Ghasemi A. Ovariectomized Rat Model of Osteoporosis: A Practical Guide. EXCLI J (2020) 19:89-107. doi: 10.17179/ excli2019-1990

34. Bhandari M, Swiontkowski M. Management of Acute Hip Fracture. N Engl J Med (2017) 377:2053-62. doi: 10.1056/NEJMcp1611090

35. Manigrasso MB, O'Connor JP. Accelerated Fracture Healing in Mice Lacking the 5-Lipoxygenase Gene. Acta Orthop (2010) 81:748-55. doi: 10.3109/ 17453674.2010 .533931

36. Li C, Zhang W, Fang S, Lu Y, Zhang L, Qi L, et al. Baicalin Attenuates OxygenGlucose Deprivation-Induced Injury by Inhibiting Oxidative Stress-Mediated 5-Lipoxygenase Activation in PC12 Cells. Acta Pharmacol Sin (2010) 31:13744. doi: 10.1038/aps.2009.196

37. Chen S. Natural Products Triggering Biological Targets-a Review of the AntiInflammatory Phytochemicals Targeting the Arachidonic Acid Pathway in Allergy Asthma and Rheumatoid Arthritis. Curr Drug Targets (2011) 12:288301. doi: $10.2174 / 138945011794815347$ 
38. Somjen D, Tordjman K, Katzburg S, Knoll E, Sharon O, Limor R, et al. Lipoxygenase Metabolites are Mediators of PTH-Dependent Human Osteoblast Growth. Bone (2008) 42:491-7. doi: 10.1016/ j.bone.2007.11.005

39. Hu S, Chen Y, Wang Z-F, Mao-Ying Q-L, Mi W-L, Jiang J-W, et al. The Analgesic and Antineuroinflammatory Effect of Baicalein in Cancer-Induced Bone Pain. Evid Based Complement Altern Med (2015) 2015:973524. doi: 10.1155/2015/973524

40. Kim JM, Lee S-U, Kim YS, Min YK, Kim SH. Baicalein Stimulates Osteoblast Differentiation Via Coordinating Activation of MAP Kinases and Transcription Factors. J Cell Biochem (2008) 104:1906-17. doi: 10.1002/ jcb. 21760

41. Kim MH, Ryu SY, Bae MA, Choi J-S, Min YK, Kim SH. Baicalein Inhibits Osteoclast Differentiation and Induces Mature Osteoclast Apoptosis. Food Chem Toxicol (2008) 46:3375-82. doi: 10.1016/j.fct.2008.08.016

42. Moura AP, Taddei SR, Queiroz-Junior CM, Madeira MF, Rodrigues LF, Garlet GP, et al. The Relevance of Leukotrienes for Bone Resorption Induced by Mechanical Loading. Bone (2014) 69:133-8. doi: 10.1016/ j.bone.2014.09.019

43. Cottrell JA, O'Connor JP. Pharmacological Inhibition of 5-Lipoxygenase Accelerates and Enhances Fracture-Healing. J Bone Joint Surg Am (2009) 91:2653-65. doi: 10.2106/JBJS.H.01844

Conflict of Interest: The authors declare that the research was conducted in the absence of any commercial or financial relationships that could be construed as a potential conflict of interest.

Copyright (C) 2021 Saul, Hohl, Franz, Meyer, Taudien, Roch, Sehmisch and Komrakova. This is an open-access article distributed under the terms of the Creative Commons Attribution License (CC BY). The use, distribution or reproduction in other forums is permitted, provided the original author(s) and the copyright owner(s) are credited and that the original publication in this journal is cited, in accordance with accepted academic practice. No use, distribution or reproduction is permitted which does not comply with these terms. 